for thoroughly disinfecting articles contaminated with the excreta of typhoid fever patients, and that the nurses at general hospitals ought to be thoroughly instructed as to the absolute necessity of completely disinfecting such articles. 2. The nursing staff at "fever" hospitals have been proved to be less prone to take typhoid fever than those at general hospitals. I would answer that serious epidemics occurring in the nursing staff at general hospitals prove that the drains of those hospitals are defective. 3. The custom of admitting typhoid fever patients to "fever" hospitals has obtained for a great number of years, and it is undesirable to alter it, as comparatively few beds are occupied by these patients. The reply is that though it is true in each "fever" hospital the number of beds allotted to typhoid fever is comparatively small, yet if all the "fever" hospitals are taken together it will be found that in the aggregate a large number of beds are occupied by typhoid fever patients which might have been utiliséd for really "infectious" diseases, such as scarlet fever. One other point that has been argued is that as typhoid fever has to be "notified" it ought to be scnt to a "fever" hospital, but erysipelas and puerperal fever have also to be "notified" and they are not sent to fever hospitals. I feel sure that if the ratepayers are obliged to pay enormou. sums for the "fever" hospitals they will certainly give less to the voluntary hospitals.

I am, Sirs, yours faithfully,

Blackheath, Aug. 8th, $1896 . \quad$ ROBERT S. WAINEWriGHT.

\section{"THE POSSIBILITY OF OVARIAN PREGNANCY."}

To the Editors of THE LANCET.

SIRS,--I am writing away from references, and, therefore cannot give the date of my most recent papers bearing on this subject, but they are in the Transactions of the Royal Medical and Chirurgical Society some four or five years ago, and I am somewhat disappointed that they should have escaped the notice of one so skilled in gynæcological literature as Mr. Alban Doran. In these papers are given at length the reasons why such cases as that published by Dr. Oliver are to be classed as broad ligament pregnancies, and three similar have been lately described, two by myself and one by Dr. Heywood Smith. I have still to say what I said fifteen years ago that pregnancy developing in the ovary may be possible, but it has yet to be proved.

$$
\text { I am, Sirs, yours truly, }
$$

The Crescent, Birmingham, Aug. 8th, 1896. LAWson TAIT.

\section{THE NOMENCLATURE OF DISEASES.}

\section{To the Editors of THE LANCET.}

SIRS,--In the "suggestions" respecting the filling in of death certificates to be found on the first page of the usual book of forms will be found the following words: "State the causes of death in terms as precise as possible and use the names adopted in the nomenclature of the Royal College of Physicians." This suggestion I make it a matter of duty to follow as closely as I can ; consequently I am disappointed to find that the third edition of the "Nomenclature" just published has done nothing to help one over a difficulty which is often met with in filling up a death certificate in a case like the following, which is no uncommon one. A child aged three months was brought to me because it "did not seem to be getting on, but was falling away." The child was illegitimate and had been nursed by its mother for one month, after which it was put out to be nursed and was fed upon condensed milk, biscuits, \&c. From that time the child wasted to mere skin and bone, and died the day after I saw it. The cause of death was simple starvation from deprivation of breast-milk and inability to digest artificial food. How was the death certificate to be filled in?

"Starvation" (p. 13 in the "Nomenclature"), including, as it does, criminal neglect (which was absent in this case), would not have been accepted by the Registrar, especially in the case of an illegitimate child, without further inquiries, and, perhaps, a coroner's inquest, besides being a term which would naturally be resented by the relatives who, unfortunately, see the certificate. "Inanition" (p. 428) is "not to be employed in the registration of disease," and is as objectionable as "starvation" and for similar reasons. "Marasmus" (p. 437) is a term no longer used. "Debility" (p. 17) is too vague, and when the cause is known (as it is in this particular case) it should be "returned under the head of the primary cause." I can find no such heading, nor one which is in the least degree applicable to the case.

The tables drawn up by the Society of Medical Officers of Health are much better in this respect, since they include under the head of Dietetic Diseases (Table 3) "want of breast milk." In this instance I filled up the certificate as follows: Primary, deprivation of breast-milk and inabilits to digest artificial food; secondary, debility and atrophy. I remain, Sirs, yours faithfully,

Aug. 8th, 1896. R. BRUCe Fercuson, M.A., M.D. Cantab.

\section{"IHE BRITISH MEDICAL BENEVOLENT SOCIETY:"}

To the Editors of THE LANCE'T.

Sins,-I have to pay over a legacy of $\$ 50$ under a will to a society described in the will as "The British Medical Benevolent Society." Can any of your readers kindly inform. me, through the medium of your valuable paper, whether there is or has been in existence a society bearing that precise title, and if so what is the address or office of the society? The nearest to it which I find is the British Meadical Benevolent Fund.--I am, Sirs, your obedient servant,

$$
\text { Augr. 11th, } 1896 .
$$

COUNTRY SOLICITOR.

*** We believe that the British Medical Benevolent Func and the Royal Medical Benevolent College have before now put in claims to the same legacy where the vague wording of the will had left it in doubt which charity it had beer intended by the testator to benefit.-ED. L

\section{"WHY IS THE LEFT HEART STRONGER THAN THE RIGHT?"}

To the Editors of THE LANCET.

SrRS, - -Dr. Alexander Morison contends that the greater re sistance in the systemic as compared with the pulmonary segment is essentially due to (1) the greater length of the former, and (2) to the greater quantity of blood it contains. It is not difficult to show that both these contentions are wrong. 1. That mere length of circuit takes little part in causing resistance to the blood-flow is obvious from many considerations: (a) The length of the systemic circuit is chiefly determined by the length of the larger arteries, such as the aorta, the brachials, and the femorals. Now while there can be little doubt that long, narrow arteries like the spermatic offer considerable resistance, such is not the case with large arteries. This fact, which I have myself experimentally demonstrated, is acknowledged by most authorities. $H$. Jacobson long ago pointed out that "the pressure in the principal arterial trunks remains very nearly identical with that of the bulbus aortæ," and more recent authorities insist upon the same fact. Blood pressure falls very little along such an artery as the brachial. (b) If mere length of circuit plays a large part in causing resistance the systemic veins of such animals as the whale and the giraffe would offer a great resistance; buts. they do not. (c) Further, on this assumption, we should expect to find a greater disproportion between the two sides. of the heart in animals like the giraffe, which possess a lon: systemic circuit in proportion to their weight than obtains in such an animal as the rabbit, for instance; but I am not. aware that any such disproportion exists. (d) Nothing more conclusively shows the small influence of length of circuit. on resistance than the comparatively small difference in. the systemic resistance, as determined by carotid pressure, in animals differing greatly in size. Thus while the carotid pressure of the horse varies from 160 to $200 \mathrm{~mm}$. Hg., that of the sheep, with a much shorter circuit, varies between 155 to $210 \mathrm{~mm}$. Hg. (Langely of Cambridge). 2. Dr. Morison's contention that the larger quantity of blood in the systemic as compared with the pulmonary circuit helps to make resistance greater in the former is even more easily disposed of. Paradoxical as it may seem, it is the very opposite of the truth. In so far as this larger quantity of systemic blood is the outcome of the larger systemic capacity it is actually a reason why systemic resistance should be less than pulmonary. How greatly Dr. Norison is in error here 